\title{
IMPACT OF POSITIONING TRAINING ON THE QUALITY OF MAMMOGRAPHY
}

Henrique Lima Coutoํㅜ, Tereza Cristina Ferreira de Oliveira', Julia Grichtolik Cantagalli Paiva', Kelly de Jesus Medeiros' ${ }^{1}$ Vivian Paula Barbosa Paes ${ }^{1}$

${ }^{1}$ Redimama, Centro de Referência no Diagnóstico Mamário - Belo Horizonte (MG), Brazil.

Introduction: Mammography (MMG) reduces breast cancer mortality. Positioning is a critical factor for the quality of the test. Objective: To evaluate a positioning training program (PTP) to improve MMG quality. Methods: This is a case-control study of an MMG PTP. This study was performed in a private service specialized in breast diagnosis. We evaluated 200 projections from 50 MMGs carried out by two experienced technicians (6 years and 17 years of practice) - 25 screenings each. Performance criteria were evaluated in the mediolateral oblique (MLO) and craniocaudal (CC) views. In $\mathrm{CC}$, we considered adequate positioning a good projection of outer quadrants $(\mathrm{OQ})$, visualization of the pectoralis major (PM), centered nipples (CN), good projection of inner quadrants (IQ), no folds or creases, nipples in profile, and symmetrical breasts (SB). The low positioning of the Bucky was considered an error criterion. In the MLO evaluation, the criteria for an adequate positioning were the visualization of inframammary angles (IMA), nipples in profile and at the PM level, SB, no folds or creases, and symmetrical PM. Hanging breasts and visualization of the pectoralis minor (Pm) were considered positioning failures. An 11-hour theoretical and practical training was provided: 7 hours of practice and 4 hours of theory; new tests were performed, and the quality criteria were evaluated. Results: Positioning errors significantly decreased after the PTP. CC errors dropped from 39\% to 11\%. MLO errors decreased from 36\% to $13 \%$. After the PTP, the CC criteria evaluated improved: good projections of OQ - from $50 \%$ to $94 \%$; visualization of the PM - from $21 \%$ to $62 \%$; $\mathrm{CN}$ - from $49 \%$ to $79 \%$; good projections of IQ - from $45 \%$ to $100 \%$; lack of folds or creases - from $74 \%$ to $88 \%$; nipples in profile - from $91 \%$ to $95 \%$; SB - from $86 \%$ to $98 \%$. The low positioning of the Bucky dropped from $19 \%$ to $0 \%$. The MLO criteria evaluated also improved after the PTP: visualization of IMA - from $45 \%$ to $82 \%$; nipples in profile - from $93 \%$ to 95\%; nipples at the PM level - from $24 \%$ to $84 \%$; lack of folds or creases - from $39 \%$ to $70 \%$; SB - from $90 \%$ to $100 \%$; symmetrical PM - from $56 \%$ to $82 \%$; symmetrical nipples - from $72 \%$ to $86 \%$; visualization of the Pm - from $13 \%$ to $7 \%$. Conclusions: The MMG PTP improved the quality of the test, the gold standard in the early detection of breast cancer. PTP acts in a vulnerable part the human. The results indicate that a simple and low-cost intervention of low technological complexity can significantly affect the quality of MMGs and screening programs in our country. 